ORIGINAL ARTICLE

\title{
Observational study of suspected maltreatment in Italian paediatric emergency departments
}

\author{
S Palazzi, G de Girolamo, T Liverani, on behalf of IChilMa (Italian Child Maltreatment \\ study group)
}

Arch Dis Child 2005;90:406-410. doi: 10.1136/adc.2003.040790 See end of article for
authors' affiliations

Correspondence to: Dr S Palazzi, Newcomen Centre, Guy's Hospital, London SE1 9RT, UK; sp651@columbia.edu

Accepted 6 August 2004

\begin{abstract}
Aims: To evaluate how often children seen in paediatric accident \& emergency (A\&E) departments were suspected of abuse or neglect, and to explore some of the correlates of suspected child maltreatment. Methods: Multicentre, cross-sectional study of 15 randomised census days during a six month period. Trained research assistants working with local paediatric staff completed a purpose made anonymised checklist covering sociodemographic and medical information. A six point suspicion index was used to rate compatibility with child maltreatment based on the occurrence of observable harm. Statistical analysis was carried out on the basis that a score of 4 or more was suspicious of child maltreatment. Nineteen hospitals provided standardised paediatric A\&E consultation data on 0-14 year olds presenting between 10 am and $10 \mathrm{pm}$.

Results: Of 10175 assessed children, 204 aroused suspicion of child maltreatment $(95 \% \mathrm{Cl} 163$ to 214 per 10000$)$. In a logistic regression model of suspected maltreatment statistically significant associations were found with socioeconomic disadvantage, children living in single parent families, and developmental delay. There was no correlation with pre-school age, male gender, foreign origin, or living in urban areas. Conclusions: Child maltreatment based on immediate scoring of suspicion, focused on observable harm, occurred in $2 \%$ of a representative sample of paediatric emergency consultations in Italy. This was more common if there were associated social and developmental vulnerabilities. True prevalence of child maltreatment in emergency departments remains elusive because of changing definitions and forensic validation problems.
\end{abstract}

\section{METHODS}

\section{Sample and assessment}

The study included 19 hospital A\&E departments ranging from large teaching hospitals to medium sized suburban hospitals. There were 13 general hospitals and six specialist children's hospitals. The annual flow of A\&E paediatric consultations ranged from about 5000 per year at Vimercate Hospital, Milan, to over 50000 at Santobono Hospital, Naples. In Italy all children attending A\&E departments are seen by paediatric medical staff before leaving the hospital.

Research assistants were trained to complete a screening checklist summarising the clinical notes of all children up to age 14 seen in A\&E for any reason between 10 am and $10 \mathrm{pm}$, on 15 days randomly selected by computer over a six month period, done in such a way that any weekday occurred at least twice. Information was sought from the accompanying adult(s) and completed by direct clinical observation. The checklist included the items shown in tables 1 and 2. Child protection agencies were expected to be alerted in relation to cases that aroused suspicion of maltreatment independent of the study procedures. Complete physical examinations were performed whenever possible, especially in younger children.

\section{Index of suspicion}

The main outcome variable was a six point ordinal scale assessing the possibility of child maltreatment, defined as probable or definite child physical or sexual abuse or severe neglect. A concept of compatibility was used, rather than risk assessment, so that any confusion with statistical language was avoided.

The compatibility measure ranged from 1 (no suspicion) to 6 (definite child abuse or neglect). Local child health teams made their assessments in collaboration with the research assistants and jointly attributed the score. Clinicians did not know that 1-3 would be considered to be negative, and 4-6 positive for suspicion of maltreatment in the statistical analysis. Different suspicion thresholds would obviously yield different prevalence estimates (not necessarily different correlations). 
Table 1 A\&E checklist items by compatibility with child maltreatment

\begin{tabular}{|c|c|c|c|c|c|c|c|}
\hline & \multicolumn{3}{|c|}{ Positive (suspicion score 4-6) } & \multicolumn{3}{|c|}{ Negative (suspicion score 1-3) } & \multirow[b]{2}{*}{$\chi^{2} \mathrm{p}$} \\
\hline & $\begin{array}{l}\text { Assessed for } \\
\text { the variable (n) }\end{array}$ & $\begin{array}{l}\text { Positive for the } \\
\text { variable (n) }\end{array}$ & $\%$ & $\begin{array}{l}\text { Assessed for } \\
\text { the variable (n) }\end{array}$ & $\begin{array}{l}\text { Positive for the } \\
\text { variable (n) }\end{array}$ & $\%$ & \\
\hline Consultations made on census dates & 204 & 190 & 93.1 & 9933 & 9056 & 91.2 & NS \\
\hline Consultations made in weekends or holidays & 204 & 68 & 33.3 & 9931 & 3898 & 39.3 & NS \\
\hline Afternoon consultations & 127 & 61 & 48.0 & 4684 & 2440 & 52.1 & NS \\
\hline Delay of 3 or more days before consultation & 175 & 26 & 14.9 & 7824 & 987 & 12.6 & NS \\
\hline $\begin{array}{l}\text { Other A\&E consultations in previous } \\
12 \text { months }\end{array}$ & 188 & 44 & 23.4 & 9498 & 1346 & 14.2 & $<0.001$ \\
\hline Male gender & 204 & 121 & 59.3 & 9926 & 5668 & 57.1 & NS \\
\hline Age up to 4 years & 199 & 117 & 58.8 & 9493 & 5457 & 57.5 & NS \\
\hline Born before 36 th week of gestation & 185 & 6 & 3.2 & 9200 & 278 & 3.0 & NS \\
\hline Single parent family & 202 & 44 & 21.8 & 9762 & 543 & 5.6 & $<0.001$ \\
\hline Foreign nationality & 145 & 25 & 17.2 & 9297 & 791 & 8.5 & $<0.001$ \\
\hline Socioeconomic disadvantage & 200 & 92 & 46.0 & 9688 & 1447 & 14.9 & $<0.001$ \\
\hline Urban residence ( $>15000$ inhabitants) & 203 & 172 & 84.7 & 9873 & 8308 & 84.1 & NS \\
\hline $\begin{array}{l}\text { History of contacts with social or mental } \\
\text { health services }\end{array}$ & 192 & 27 & 14.1 & 9278 & 320 & 3.4 & $<0.001$ \\
\hline Anomalous behaviour at examination & 202 & 41 & 20.3 & 9809 & 186 & 1.9 & $<0.001$ \\
\hline Significant growth delay & 202 & 15 & 7.4 & 9811 & 98 & 1.0 & $<0.001$ \\
\hline Development (cognitive/emotional) delay & 201 & 14 & 7.0 & 9783 & 90 & 0.9 & $<0.001$ \\
\hline Episode defined as an accident & 190 & 71 & 37.4 & 9349 & 2119 & 22.7 & $<0.001$ \\
\hline Non sport accident & 61 & 58 & 95.1 & 2062 & 1942 & 94.2 & NS \\
\hline
\end{tabular}

\section{Informed consent}

The data were anonymised at an early stage of the study process. The ethical committees of the relevant institutions fully evaluated the research protocol. The study panel was told that, according to the Italian laws, they did not deem necessary a formal approval because individuals' names were not filed or processed electronically in any way, and information concerning the identity of the patients was not stored.

\section{Statistical analysis}

Data were collected on single sheet forms, then captured as dichotomous variables and processed using Microsoft Access and SPSS and STATA 5 software. $\chi^{2}$ tests were applied to compare suspected positives and negatives (with Yates's correction for small frequencies). The association between number of patients and number of positives was examined with parametric and non-parametric tests to assess whether patients' volume influenced suspicion rate. A multivariate analysis was used to further explore how positives and negatives differed in terms of maltreatment risk. Odds ratios were derived for each sociodemographic characteristic associated with child maltreatment. The logistic model was run on STATA 5, entering seven variables in the following order: age up to 4, male gender, foreign nationality, urban residence, socioeconomic disadvantage, single parent family, and development (cognitive and/or emotional) delay.

\section{RESULTS}

\section{Sample characteristics and missing data analysis}

The study took place between April and September 2000. Pooling the 15 index days, the total number of consultations that took place in the participating A\&E departments was 10 955. This sample was assumed to be approximately representative of the 264000 A\&E paediatric consultations occurring in the 19 participating hospitals in the year. About $50 \%$ of the actual sample was enrolled in June and July (summer school term ends by mid-June in Italy). Ninety two per cent of consultations occurred on the randomised calendar dates, while the remainder were recorded on alternative (but same weekday) dates to make up for unanticipated local key staff days off. Overall, weekend consultations accounted for $39.1 \%$ of the sample. The largest number of consultations was provided by the Children's Hospital Santobono, Naples ( $\mathrm{n}=2160$ ), accounting for $19.7 \%$ of the sample. Two cities, Naples in the south (two hospitals), and Turin in the north, contributed one third of the sample. Remaining cities, ranked by sample size, included Rome (two

Table 2 A\&E checklist items by compatibility with child maltreatment (continued)

\begin{tabular}{|c|c|c|c|c|c|c|c|}
\hline & \multicolumn{3}{|c|}{ Positive (suspicion score 4-6) } & \multicolumn{3}{|c|}{ Negative (suspicion score 1-3) } & \multirow[b]{2}{*}{$\chi^{2} p$} \\
\hline & $\begin{array}{l}\text { Assessed for the } \\
\text { variable (n) }\end{array}$ & $\begin{array}{l}\text { Positive for the } \\
\text { variable (n) }\end{array}$ & $\%$ & $\begin{array}{l}\text { Assessed for the } \\
\text { variable (n) }\end{array}$ & $\begin{array}{l}\text { Positive for the } \\
\text { variable (n) }\end{array}$ & $\%$ & \\
\hline Skin lesions possibly traumatic & 194 & 75 & 38.7 & 9316 & 1102 & 11.8 & $<0.001$ \\
\hline Oral lesions possibly traumatic & 196 & 8 & 4.1 & 8941 & 115 & 1.3 & $<0.001$ \\
\hline Genital or perineal lesions possibly traumatic & 143 & 4 & 2.8 & 5921 & 22 & 0.4 & $<0.001$ \\
\hline Present or past fractures & 191 & 14 & 7.3 & 9491 & 221 & 2.3 & $<0.05$ \\
\hline Present or past burns & 192 & 4 & 2.1 & 9423 & 60 & 0.6 & $<0.001$ \\
\hline Present or past head trauma & 193 & 18 & 9.3 & 9413 & 307 & 3.3 & $<0.001$ \\
\hline Discharge diagnosis completed & 204 & 195 & 95.6 & 9933 & 9613 & 96.8 & NS \\
\hline Hospitalised following consultation & 195 & 12 & 6.2 & 9798 & 589 & 6.0 & NS \\
\hline History contradictory with clinical presentation & 201 & 49 & 24.4 & 9912 & 125 & 1.3 & $<0.001$ \\
\hline Circumstances deemed preventable & 200 & 147 & 73.5 & 9881 & 437 & 4.4 & $<0.001$ \\
\hline Two or more clinicians involved in the evaluation & 203 & 194 & 95.6 & 9894 & 8591 & 86.8 & $<0.001$ \\
\hline
\end{tabular}


Table 3 Explanatory model of paediatric suspicion of child maltreatment in emergency evaluations

\begin{tabular}{lll}
\hline & Odds ratio & $95 \% \mathrm{Cl}$ \\
\hline Age up to 4 years & 0.99 & 0.69 to 1.41 \\
Male gender & 1.1 & 0.77 to 1.56 \\
Foreign nationality & 1.37 & 0.84 to 2.25 \\
Urban residence (>15 000 inhabitants) & 1.35 & 0.77 to 2.35 \\
Socioeconomic disadvantage* & 4.76 & 3.31 to 6.85 \\
Single parent family* & 2.55 & 1.56 to 4.17 \\
Development (cognitive/emotional) delay* & 5.39 & 2.5 to 11.61 \\
\hline
\end{tabular}

Risk of suspicion expressed as odds ratios and 95\% confidence intervals $(n=8624)$.

*Statistically significant association within the model (pseudo $r^{2} 0.09, \operatorname{LR} \chi^{2} 122.52, p<0.001$ ).

hospitals), Milan (three hospitals), Genoa, Bari, Ancona, Trieste, Alessandria, Padoa, Rimini, Bologna, Modena, Cagliari, and Novara.

The outcome variable (that is, maltreatment compatibility score) was missing in 780 cases $(7.1 \%)$, mainly due to high numbers of emergency cases, making reliable scoring impossible. Consequently, the number of valid evaluations was 10175 . There were 751 (7.4\%) consultations in which joint evaluations of the outcome variable by paediatricians working in A\&E and research assistants were not possible due to high caseloads. There was no statistically significant sociodemographic difference between the excluded patients and the remainder of the sample.

The mean age (SD) of the patients was 4.8 (3.9) years. However, 57\% of consultations were for children under 4 . Fifty seven per cent were males. The distribution of suspected maltreatment varied significantly between hospitals (fig $1, \chi^{2}$ $\mathrm{p}<0.001)$.

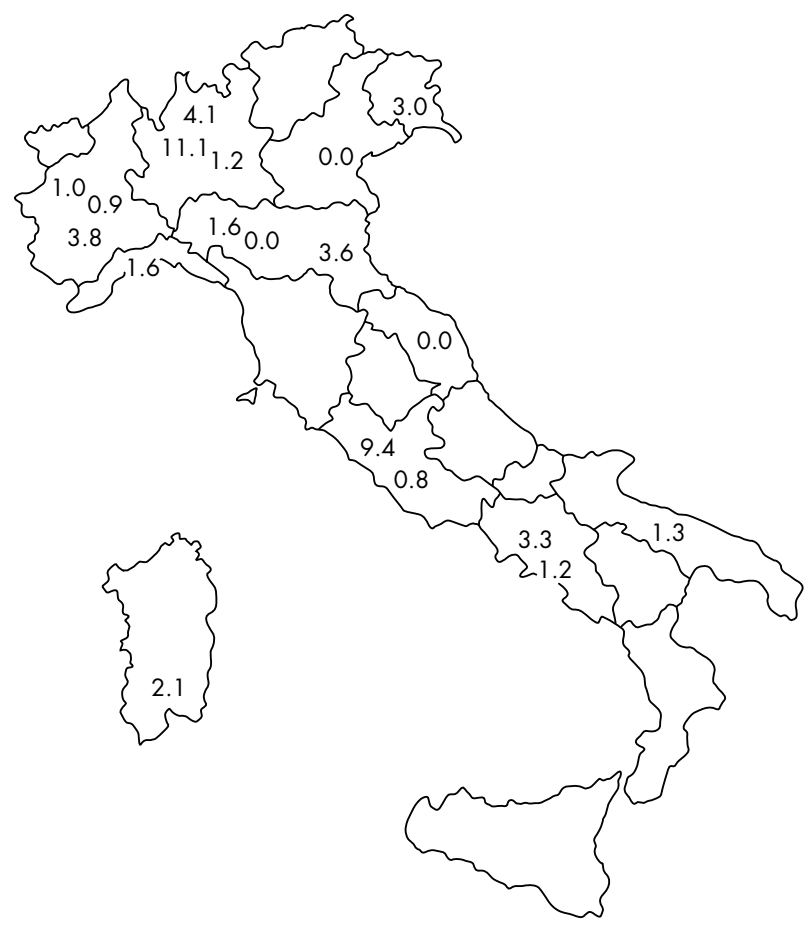

Figure 1 Child maltreatment suspected by paediatric emergency staff (\%). Approximate locations of participating hospitals and ratios of children scoring 4 or above in "compatibility with the concept of child abuse or neglect, understood as physical, sexual, or mental harm (i.e. non-subjective evidence of injury suffered because of others, known or unknown)". Scale: 1, ruled out; 2, almost excluded; 3, very dubious; 4, dubious; 5, almost certain; 6, certain. Clinicians did not know what threshold was to be set.

\section{Frequency of suspected child maltreatment}

The maltreatment compatibility score showed a skewed distribution, with 9426 scoring one, 369 scoring two, 142 scoring three, 125 scoring four, 47 scoring five, and 32 scoring six. There were 204 consultations $(2 \%)$ indicating maltreatment based on a score of 4 or more. There was no significant difference in suspicion frequency between census and catchup dates (table 1). Including missing data patients in the overall denominator, the point estimate translates into a $95 \%$ confidence interval of 163 to 214 suspect victims per 10000 consultations per year.

Figure 1 shows hospital positive ratios on a drawing of the country, ranging from $0 \%$ to $11.1 \%$. There were statistically significant differences between hospitals $\left(\chi^{2} \mathrm{p}<0.001\right)$. Local sample sizes, however, were not significantly associated with positives frequencies.

Table 1 compares the sociodemographic characteristics of those scoring positive for suspected maltreatment against the remaining sample. These characteristics included male gender, nationality, socio economic status, one parent family, and residence in towns with over 15000 inhabitants. All these variables, except male gender and urban residence, were significantly more frequent in suspect cases $(\mathrm{p}<0.001)$.

Higher frequency of suspicion was significantly associated with A\&E attendance in the previous year, the episode commonly being an accident and/or a history of contact with family support teams or child mental health services $(\mathrm{p}<0.001)$.

Manifestations that were statistically more frequent in suspicious cases included anomalous behaviour at examination, clinically significant growth delay, and developmental delay $(\mathrm{p}<0.001)$.

Table 2 details the proportions of skin, oral, and genitoperineal lesions of possible traumatic cause, as well as evidence of present or past fractures, burns, and head trauma. Except for burns $(\mathrm{p}<0.05)$, suspicion distribution was statistically significant for all these characteristics at the 0.001 level.

The three most frequent A\&E presentations were fever, head trauma, and inguinal pain. Following paediatric assessment about $6 \%$ of children were admitted to hospital wards in both groups. Medical histories were felt more often incongruous in children with suspicion of maltreatment than in the remaining sample $(\mathrm{p}<0.001)$. Similarly, circumstances relating to the reason of consultation were considered preventable or due to carers' unawareness, imprudence, or insufficient supervision of the child more often in suspicious cases $(p<0.001)$. Of the 165 of 200 suspected cases where evaluation was possible, $82.5 \%$ were ascribed to neglect and the remainder as due to sexual or physical abuse.

\section{Clinical correlates of child maltreatment}

The outcome variable was not derived from other items, and its correlations were explored by multivariate analysis based 


\section{What is already known on this topic}

- Judicial statistics underestimate child maltreatment

- Self-report surveys may overestimate child abuse and neglect due to recall and other methodological biases

- Clinicians are at variance in sensitivity to child maltreatment

on 8624 records ( 1551 excluded due to missing data). The analysis included age, gender, nationality, urban versus rural residence, social disadvantage, family composition, and developmental delay. The model's overall variance was statistically significant (table 3). Age, gender, nationality, and living in urban areas were not statistically significantly associated with suspicion of maltreatment in the multivariate model (whereas the association with nationality appeared significant in the bivariate statistic of table 1). However, socioeconomic disadvantage, single parent family status, and cognitive or emotional delay were significantly associated with likelihood of maltreatment suspicion even after controlling for age, gender, nationality, and residence.

\section{DISCUSSION}

Independent of the aims of the study, child protection agencies were alerted to the 32 (of 10 175) cases that scored highest for child maltreatment based on observable clinical harm. This indicates a formal maltreatment-allegation rate five times higher than the rate found in a retrospective survey of clinical records in 68 A\&E units across 16 Italian regions in 1998, which was 6 per 10000 (252/410 566). ${ }^{2}$

Based on the clinical suspicion threshold set in the current study, the observed $2 \%$ is intermediate between those reported in other A\&E surveys carried out in other countries, which yielded rates of 60 per $10000,{ }^{3} 1.3 \%,{ }^{4}$ and $4 \%{ }^{5}$ (the former study considered children up to 2 years of age; the latter two studies only included children referred for trauma).

Besides population variability, higher A\&E alleged maltreatment rates could be explained by participation in research and using flowchart-style reminders in the assessment notes, to help increase the awareness, consideration, and documentation of injuries to children. ${ }^{6}$ Audit has been shown to increase staff awareness of the possibility of child abuse. ${ }^{7}$ In nine A\&E surveys from different countries, mainly based on notes reviews, the median rate of maltreatment was 110 per 10000 (sample sizes ranging from 642 to 26779 , median 11066$){ }^{8-15}$ The suspicion rate exposed in our study is twice that.

This study was unique in getting clinicians to record how many children had suspected maltreatment, and why, before allegations were made and child protection schemes activated. It is an important contribution to the epidemiology of child maltreatment. The study showed a suspected maltreatment rate of 200 per 10000 consultations (95\% CI 163 to 214). With a definition stressing the concept of compatibility with abuse or neglect, and the occurrence of observable clinical harm, 1 in 50 children were suspected of maltreatment. This is a major challenge for emergency departments.

The study confirms and reinforces that developmental delay and incongruity between observed injuries and the history are significantly associated with suspicion of maltreatment in the A\&E paediatric setting. Hospital effect was not included in the multivariate model of suspicion because the study focused on patient related characteristics. Clinicians' variability in addressing child protection issues is well recognised. ${ }^{6}$
What this study adds

- Two per cent of consultations in Italian emergency departments aroused clinical suspicion of child maltreatment

- Children's age, gender, living in urban residence, and nationality were not linked to suspicion of child maltreatment

- Child maltreatment was more likely to be suspected along with developmental delay when accounting for sociodemographic factors.

\section{Study limitations}

During the preparatory stage of the study many methodological problems were addressed, including the validity of the outcome measure and the reliability of the checklist. Wording was painstakingly reviewed, and terms such as risk (in a nonstatistical sense) dropped in favour of compatibility and suspicion. The six point scale of compatibility with maltreatment did not measure child maltreatment per se, but rather reflected clinicians' perceptions during routine assessments. Its credibility and validity can be inferred from its correlation to sociodemographic and clinical characteristics known to be associated with maltreatment.

The representativity of the study is limited to children attending A\&E in the selected hospitals. Training and local discussion of implementation was used to reduce variability in scoring. This was further reduced by research assistants and local paediatricians jointly agreeing the compatibility score, where possible.

Not all consultations could involve full physical examinations, particularly in older children. More meticulous assessments would not necessarily lead to higher suspicion counts. In any case they are unachievable in hectic A\&E settings. The statistical analysis had to deal with missing data compromising between using available information and minimising spurious associations.

It is of note that developmental (cognitive and/or emotional) delay was the overall clinical characteristic most correlated with suspected maltreatment. However, maltreatment may cause developmental delay, or developmentally delayed children may bring their (often poor and single) parents or carers to the end of their tether.

\section{Conclusions}

Emergency departments play a pivotal role in detecting child maltreatment. This paper provides epidemiological information about the positive predictive value of a screening programme for child maltreatment in a defined setting, that is the occurrence of suspected child abuse and neglect based on clinicians' perceptions of compatibility with maltreatment using a simple score. A $2 \%$ suspicion rate may or may not reflect how many children are indisputably victims of maltreatment within an exact clinico-legal definition. Suspected cases were often characterised by delayed development, social disadvantage, and single parent household. The high incidence of suspected child maltreatment in emergency departments presents both a challenge and an opportunity to public authorities responsible for protecting children.

\section{ACKNOWLEDGEMENTS}

Dr Nandu Thalange, consultant paediatrician at Norwich and Norfolk University Hospital, provided insightful editorial comments. 


\section{Authors' affiliations}

S Palazzi, Michael Rutter Centre for Children and Adolescents,

Maudsley Hospital, London, UK

G de Girolamo, T Liverani, National Mental Health Project, National Institute of Health, Rome, Italy

The study was funded by the Italian NIH in the framework of the National Mental Health Project

Competing interests: none declared

The IChilMa (Italian Child Maltreatment study group) included, in alphabetical order by city: D Besana, S Susigan (Azienda Ospedaliera Santi Antonio Biagio e Cesare Arrigo, Alessandria); G Cardoni, L Palma (Ospedale Pediatrico Salesi, Ancona); P Maremonti, D Intrano (Ospedale Giovanni XXIII, Bari); GP Salvioli, R Alessandroni, S Galletti (Pediatric Clinic, University of Bologna); C Pintor, M Concas (Pediatric Clinic, University of Cagliari); O Ciccone, L Cresta, F Gargiulo (Ospedale Gaslini, Genoa); V Carnelli, G Rollandi (Pediatric Clinic " $G$ De Marchi", University of Milan); G Chiumello, A Flores D'Arcais, I Coppa (Pediatric Clinic, Ospedale S Raffaele, Milan); G Rondanini, M Pirrotta, (Ospedale di Vimercate, Milan); E Caffo, S Bernasconi, S Madeo (Child Neuropsychiatry Institute and Pediatric Clinic, University of Modena and Reggio Emilia); M Berni Canani, N Aragione (Ospedale Santobono, Napoli); P Siani, C Miniero (Ospedale Cardarelli, Napoli); G Bona, R Osello, (Pediatric Clinic, University of Piemonte Orientale "A Avogadro", Novara); P Facchin, S Manea (Pediatric Clinic, University of Padua); M Castello, E Properzi, R Guidi, F Mizzoni (Pediatric Clinic, University "La Sapienza", Rome); G Viviano, A Musolino, M Marano, M Mattina (Ospedale Bambin Gesù, Rome); V Vecchi, F Nardocci, L Pausini, S Nucci (Department of Paediatrics, Ospedale degli Infermi, and Servizio di Neuropsichiatria Infantile, ASL di Rimini); GB Ferri, E Bordone (Azienda Ospedaliera "OIRM" S Anna, Ospedale Infantile R Margherita, Turin); A G Marchi, E Barth (IRCCS Burlo Garofolo, Trieste)

\section{REFERENCES}

1 Bardi M, Borgognini-Tarli SM. A survey on parent-child conflict resolution: intrafamily violence in Italy. Child Abuse Negl 2001;6:839-53.

2 Marchi AG, Gaeta G. I quadri clinici più frequenti dell'abuso del bambino. Riv Ital Pediatr 1999:25:696-700.

3 Rosenberg NM, Meyers S, Shackleton N. Prediction of child abuse in an ambulatory setting. Pediatrics 1982;70:879-82.

4 Pless IB, Sibald AD, Smith MA, et al. A reappraisal of the frequency of child abuse seen in paediatric emergency rooms. Child Abuse Negl 1987; 11:193-200.

5 Yamamoto LG, Wiebe RA, Matthews WJ Jr. A one-year prospective ED cohort of paediatric trauma. Pediatr Emerg Care 1991;7:267-74.

6 Benger JR, Pearce AV. Simple interventions to improve detection of child abuse in emergency departments. BMJ 2002;324:780-2.

7 Bar-on M, Zanga JR. Child abuse: a model for the use of structured clinical forms. Pediatrics 1996;98:429-33.

8 Seidel JS, Elvik SL, Berkowitz CD, et al. Presentation and evaluation of sexual misuse in the emergency department. Pediatr Emerg Care 1986;2:157-64.

9 Peclet MH, Newman KD, Eichelberger MR, et al. Patterns of injury in children. J Pediatr Surg 1990;25:85-90.

10 Christopher NC, Anderson D, Gaertner L, et al. Childhood injuries and the importance of documentation in the emergency department. Pediatr Emerg Care 1995;11:52-7.

11 Boyce MC, Melhorn KJ, Vargo G. Paediatric trauma documentation. Adequacy for assessment of child abuse. Arch Pediatr Adolesc Med 1996;150:730-2.

12 Wright MS, Litaker D. Childhood victims of violence. Hospital utilization by children with intentional injuries. Arch Pediatr Adolesc Med 1996;150:415-20.

13 Marshall WN Jr. Hospitalization of abused and neglected children. Arch Pediatr Adolesc Med 1997;151:273-5.

14 Limbos MA, Berkowitz CD. Documentation of child physical abuse: how far have we come? Pediatrics 1998;102:53-8.

15 DiScala C, Sege R, Li G, et al. Child abuse and unintentional injuries: a 10year retrospective. Arch Pediatr Adolesc Med 2000;154:9-10.

\section{IMAGES IN PAEDIATRICS}

\section{Basilar artery infarction}

A n 8 year old boy complained of headache and vomiting one morning and became drowsy in the afternoon. On the following day, diplopia and consciousness disturbance progressed. On admission to hospital, he was comatose and neurological examination revealed right hemianopsia and right ptosis. Other cranial nerve involvement was not recognised. MRI revealed T2 high intensity lesions in bilateral occipital calcarine and the left cerebellar hemisphere (fig 1). Routine laboratory examination including coagulation system, lactate, pyruvate, serum amino acid analysis, and cerebrospinal fluid analysis showed no abnormal findings. Cardiac echosonography and electrocardiography revealed no abnormal findings. Cerebral angiography revealed a basilar artery occlusion (fig 2), but no dissection of carotid arteries. Subsequently, fibrinolytic therapy was performed using urokinase; recanalisation was achieved and his consciousness level improved markedly. On the 26th day after admission he was discharged without any sequelae.

Basilar artery infarction is extremely rare in children. ${ }^{12}$ Although the causes of basilar artery infarction include
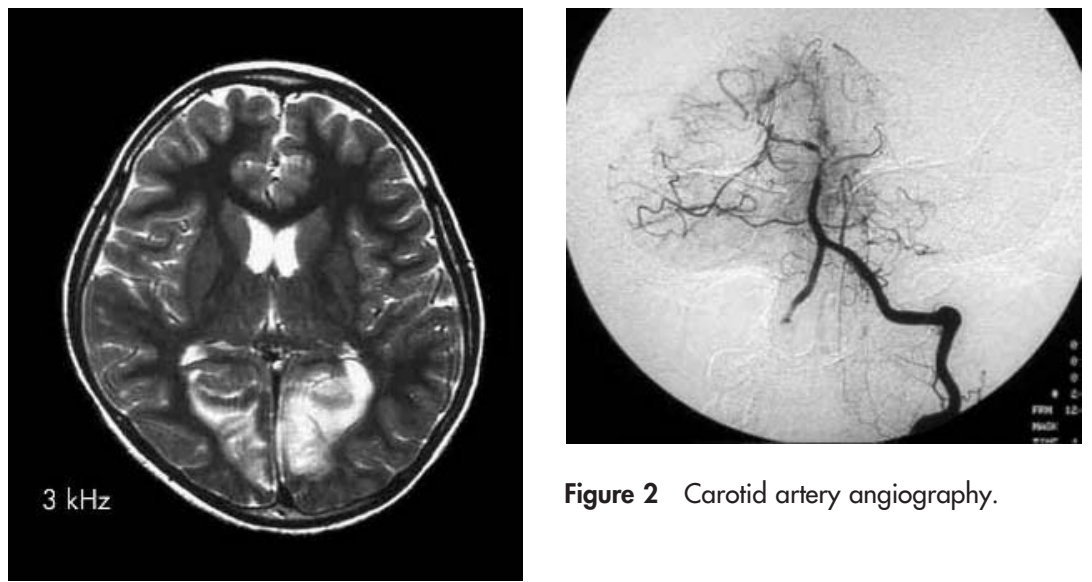

Figure 2 Carotid artery angiography.

Figure 1 T2 weighted MRI.

trauma, sepsis, endocarditis, arthritis, oral contraceptive use, hypercoagulable state, and basilar artery dissection, no apparent cause was determined in the present case, or in many other cases. Fortunately the patient recovered completely. We should be vigilant for this condition if we see a child with acute consciousness disturbance since early intervention can prevent neurological sequelae.
Department of Pediatrics, Miyagi Children's Hospital, 4-3-17 Ochiai, Aoba-ku, Sendai 989-3126, Japan; hideto@miyagi-children.or.jp

\section{References}

1 Ishikawa T, Sasaki H, Makino K, et al. Pediatric brain stem infarction caused by a basilar and vertebral artery occlusion: case report. Neurosurgery 1992;31:365-8.

2 Nakatomi H, Nagata K, Kawamoto S, et al. Basilar artery occlusion due to spontaneous basilar artery dissection in a child. Acta Neurochir 1999;141:99-104. 